\title{
SNR Performance of Various MIMO-OFDM Detectors using V-BLAST
}

\author{
Sneha Jojare \\ ME Student \\ Dept. of Electronics and Telecomm. Engineering \\ VESIT, Chembur. Mumbai University
}

\author{
Naveeta Kant \\ Professor \\ Dept. of Electronics and Telecomm. Engineering \\ VESIT, Chembur. Mumbai University
}

\begin{abstract}
The combination of multiple-input-multiple-output (MIMO) wireless technology with orthogonal frequency division multiplexing (OFDM) has been recognized as one of the most promising techniques to support high data rate, improved capacity, high performance, and reduced inter-symbol interference(ISI). The broadband channel is a typically non-lineof-sight channel and includes many impairments such as timeselective and frequency-selective fading, due to which MIMOOFDM serves as a better solution. MIMO-OFDM system has various detection techniques such as ZF (Zero Forcing), MMSE (minimum mean square error), ML (Maximum Likelihood, VBLAST (Vertical Bell Labs Layered Space-Time). V-BLAST detection undergoes SIC (successive interference calculation) technique which uses spatial multiplexing. This paper mainly focuses on SNR performances based on BER (bit error rate) of received MIMO signals by applying the V-BLAST algorithm. The algorithm was tested on ZF, MMSE, ML detectors at the receiver end of MIMO system with the help of MATLAB simulation. ML was found out to be the best configuration, and BNR improvement of $10^{2}$ and SNR of at least $12 \mathrm{~dB}$ can be easily achieved.
\end{abstract}

\section{Keywords}

MIMO-OFDM, V-BLAST, ZF, MMSE, ML, SIC

\section{INTRODUCTION}

MIMO technology has been used from over along period but new technologies needed to be developed to enable MIMO to be fully functional. New levels of processing were needed to allow some of the features of spatial multiplexing as well as to utilize some of the gains of spatial diversity. If the correlation between antennas becomes small, MIMO systems will operate effectively. As a rule of thumb, s spacing of $\lambda / 2$ (where $\lambda$ is the wavelength of the signal) is considered necessary to provide almost no correlation between the antennas [1], [16]. The combination MIMO-OFDM enables support of more antennas and larger bandwidths as it simplifies equalization dramatically. By implementing Multiple-Input Multiple-Output (MIMO) and Orthogonal Frequency-Division Multiplexing (OFDM) technologies ,data rates up to several hundreds of Mbits/s ,achieve spectral efficiencies of several tens of bits/Hz/s and diversity which are unattainable for conventional single-input single-output systems. MIMO system uses linear detection algorithms such as ZF, MMSE, V-BLAST and non-linear detection algorithm such as ML at the receiver. Many research papers explains the behavior of these detection techniques separately This paper explains how SNR performance gets better by applying V-BLAST detection algorithm on ZF,MMSE,ML detectors in detail. Vertical Bell Laboratories layered space-time (V-BLAST) detector is an efficient MIMO detection algorithm that gives better BER performance than either ZF or MMSE or $\mathrm{ML}$ at the cost of increased computational complexity.

\section{MIMO-OFDM SYSTEM MODEL}

MIMO is effectively a radio antenna technology as it uses multiple antennas at the transmitter and receiver to enable a variety of signal paths to carry the data, choosing separate paths for each antenna to enable multiple signal paths to be used. One of the core ideas behind MIMO wireless systems space-time signal processing in which time is complemented with the spatial dimension inherent in the use of multiple spatially distributed antennas, i.e. the use of multiple antennas located at different points. Between a transmitter and a receiver, the signal can take many paths. dditionally by moving the antennas even a small distance the paths used will change. Presence of various objects to the side or in between the path of transmitter and receiver, results into variety of paths. Previously these multiple paths only served to introduce interference. By using MIMO, these additional paths can be used to advantage. Thus it adds robustness to the radio link by improving the SNR, or by increasing capacity given as: $C=N M B \log 2\left(1+\frac{S}{N}\right)$ Where B is channel bandwidth, $M \& N$ number of antennas at $T x$ and $R x$ ends [9].

The two main formats for MIMO are [9],

\subsection{Spatial diversity:}

It uses two or more antennas to improve the quality and reliability of a wireless link. These two methodologies are used to provide improvements in the signal to noise ratio and they are characterized by improving the reliability of the system with respect to the various forms of fading.

\subsection{Spatial multiplexing}

It is a transmission technique which uses more than two antennas at transmitter and receiver to transmit independent and separately encoded data signals, so-called streams, from each of the multiple transmit antennas. i.e. increasing the data throughput capability.

Most of the previous work in the area of MIMO wireless has been restricted to narrowband systems. MIMO channels offers spatial diversity along with higher capacity and frequency diversity due to delay spread. Orthogonal frequency division multiplexing significantly reduces receiver complexity in wireless broadband systems. The use of MIMO technology in combination with OFDM, i.e., MIMO-OFDM therefore seems to be an attractive solution for future broadband wireless systems. OFDM provides high-speed transmission and mitigates frequency selected fading. In OFDM the high speed data streams is divided into narrowband data streams corresponding to the subcarriers. As a result the symbol duration is $\mathrm{N}$ times longer than in single carrier system with same symbol rate. OFDM has 
IFFT at transmitter and FFT at reciver which converts wideband frequency selective fading signal to narrowband flat fading signal [7],. Figure shows block diagram for MIMO-OFDM system with $M$ number of transmit antennas and $N$ number of

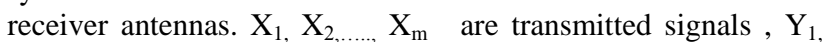
$Y_{2, \ldots}, Y_{n}$ are received signals. The received vector is given by [4][6][15],

$\widehat{\boldsymbol{y}}=\boldsymbol{H} \widehat{\boldsymbol{x}}+\widehat{\boldsymbol{n}}$

Where, $\widehat{\boldsymbol{y}}$ received vector, $\boldsymbol{H}$ is $m \times n$ channale transfer function, $\widehat{\boldsymbol{n}}$ is noise vector.

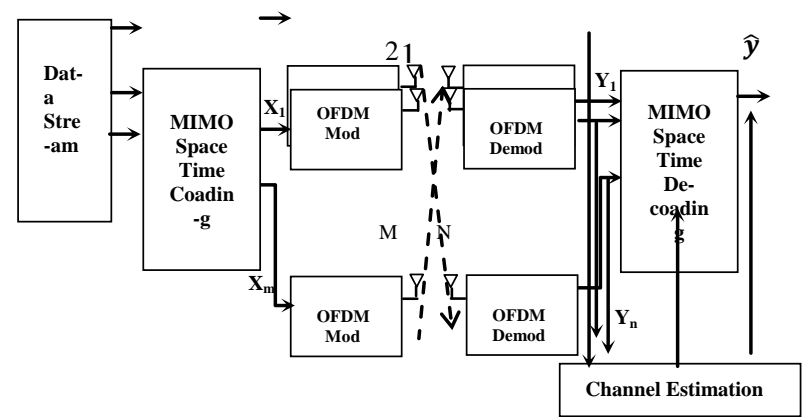

Fig 1- MIMO-OFDM System Model

\section{MIMO DETECTION TECHNIQUES}

The detection strategy is one of the prime criteria to determine the effectiveness of a communication system [15][1][11].

\section{Zero Forcing:}

Zero Forcing is a linear detection technique. The pseudo inverse of the signal is applied to the received signal in order to make a decision about one user. So the equation for filter matrix $G$ for zero forcing will be,

$$
\boldsymbol{G}=\left(\boldsymbol{H}^{\boldsymbol{H}} \boldsymbol{H}\right)^{-\mathbf{1}} \boldsymbol{H}^{\boldsymbol{H}}
$$

\section{Minimum Mean Square Error:}

Minimum Mean Square Error MMSE is also a linear detection technique but more reliable than $\mathrm{ZF}$ in case of noisy channel. MMSE does not apply pseudo inverse of signal to make decision about one user, instead it attenuates them to noise level thereby reducing the diversity order. MMSE eq will be ,

$$
\boldsymbol{G}=\left(\boldsymbol{H}^{\boldsymbol{H}} \boldsymbol{H}+\frac{\boldsymbol{N t}}{\boldsymbol{S N R}_{\boldsymbol{R}}}\right)^{-\mathbf{1}} \boldsymbol{H}^{\boldsymbol{H}}
$$

3. Maximum Likelihood:

ML is a non-linear detection technique. The BER/SNR results of ML are better than MMSE detector but at the cost of additional complexity.

\section{VERTICAL BELL LABORATORIES LAYERED SPACE-TIME (V-BLAST)}

Figure2 shows V-BLAST model for $(m \times n)$ MIMO system. . Vector encoder takes demultiplexed single stream into $M$ substreams with $\mathrm{M}$ is the number of transmitter antennas. Each substream is encoded into symbols and fed to a separate transmitter [4].

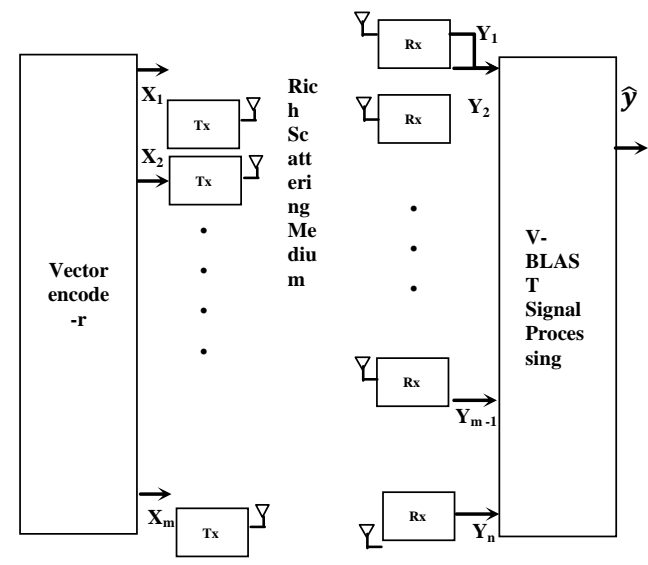

Fig 2 - V-BLAST Architecture

The modulation method in these s /stems usually is Quadrature Amplitude Modulation (QAM). QAM combines phase modulation with amplitude modulation, making it an efficient method for transmitting data over a limited bandwidth channel VBLAST's receivers operate co-channel, each receiving the signals emanating from all $\mathrm{M}$ of the transmitting antennas. Receivers $\mathrm{N}$ are, individually, conventional QAM receivers. These receivers also operate co-channel, each receiving the signals radiated from all $M$ transmit antennas [10]

\section{V-BLAST DETECTION ALGORITHM}

In V-BLAST, signal symbols are detected vertically from the same signal vector, by detecting the symbol transmitted by each transmit antenna in turn in order of decreasing estimated SNR. Figure shows steps to perform V-BLAST detection [4][9].

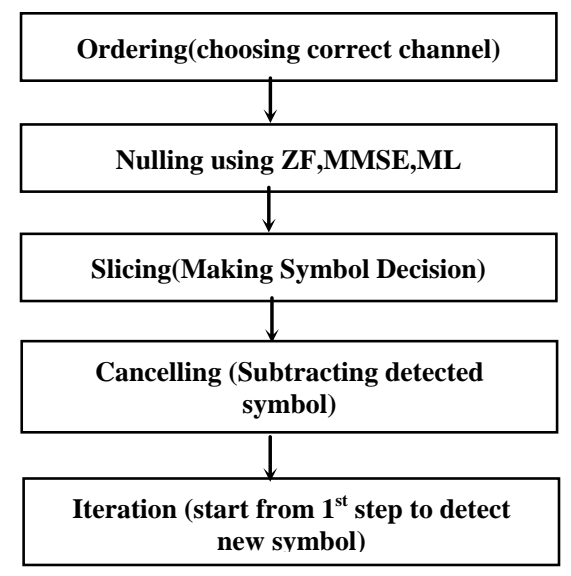

Fig 3- V-BLAST Algorithm

V-BLAST uses Sucessive Interference Cancellation (SIC) and achieves the better detection accuracy by exploiting interference cancellation. The principle of this algorithm is that the strongest (i.e., highest SNR) transmitted symbol is detected in the first step using either the ZF or MMSE or ML criteria. Then the interference from this symbol on the received MIMO signals is predicted and subtracted away to eliminate the interference of the symbol from the received signals. The same steps are repeated to detect the remaining transmitted symbols. In this way, we can cancel the interference caused by previously detected symbols to offer more accurate detection for the next detected symbol[6]. 


\section{MATLAB SIMULATION AND RESULTS}

In this paper, we have compare the SNR values Vs BER values of the received signals for QAM , ZF , MMSE , ML using VBLAST by varying number of antennas at the transmitter and receiver end. Following table shows the comparison of BER values obtained from MATLAB simulation for $12 \mathrm{db}$ SNR for SISO (Single input single output), SIMO (Single input multiple output), MISO(Multiple input single output), MIMO(Multiple input multiple output).

Table 1- Comparison of BER for 12db SNR

\begin{tabular}{|c|c|c|c|c|}
\hline \multirow{2}{*}{$\begin{array}{ll}\text { For } & \text { SNR(12 } \\
\text { Db) } & \end{array}$} & \multicolumn{4}{|l|}{ BER } \\
\hline & QAM & ZF-SIC & $\begin{array}{l}\text { MMSE- } \\
\text { SIC }\end{array}$ & ML-SIC \\
\hline $\begin{array}{l}\text { SISO }(1 \times \\
1)\end{array}$ & 0.1584 & 0.01584 & 0.0158 & 0.3981 \\
\hline $\begin{array}{l}\text { SIMO }(1 \times \\
4)\end{array}$ & 0.1584 & 0.00001 & 0.00001 & 0.00001 \\
\hline $\begin{array}{l}\operatorname{MISO}(4 \times \\
1)\end{array}$ & 0.1584 & 0.3162 & 0.1995 & 0.3981 \\
\hline $\begin{array}{l}\operatorname{MIMO}(4 \times \\
4)\end{array}$ & 0.1258 & 0.0025 & 0.0001 & 0.00001 \\
\hline
\end{tabular}

Table 2- Comparison of BER of diff MIMO systems for 12db SNR

\begin{tabular}{|l|l|l|l|l|}
\hline $\begin{array}{l}\text { For SNR(12 } \\
\text { Db) }\end{array}$ & QAM & ZF-SIC & $\begin{array}{l}\text { MMSE- } \\
\text { SIC }\end{array}$ & ML-SIC \\
\hline $\begin{array}{l}\text { MIMO }(2 \times \\
2)\end{array}$ & 0.1584 & 0.0079 & 0.00316 & 0.00158 \\
\hline MIMO $(4 \times 4)$ & 0.1258 & 0.0025 & 0.0001 & 0.00001 \\
\hline MIMO $(8 \times 8)$ & 0.1584 & 0.3162 & 0.1995 & 0.3981 \\
\hline
\end{tabular}

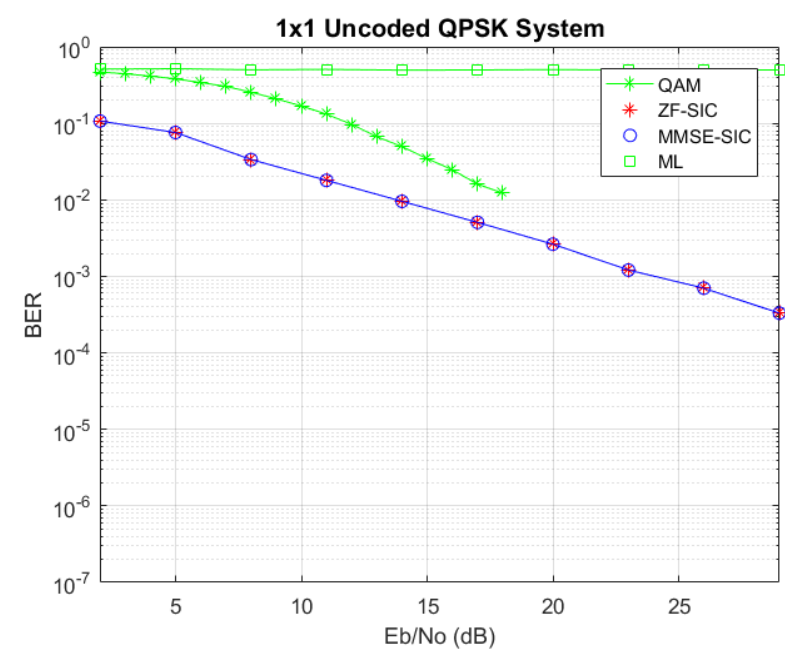

Fig 4-SNR Vs. BER plot for $(1 \times 1)$ SISO system

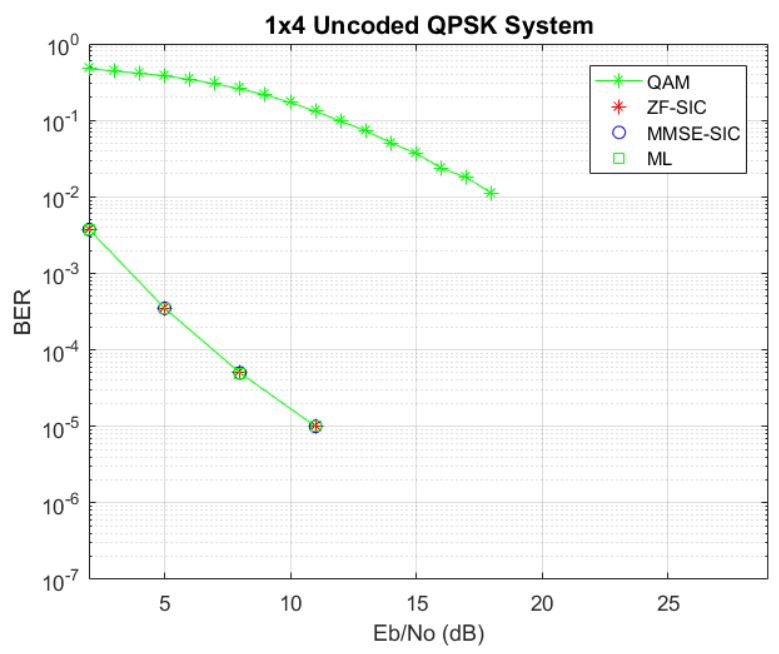

Fig 5-SNR Vs. BER plot for $(1 \times 4)$ SIMO system

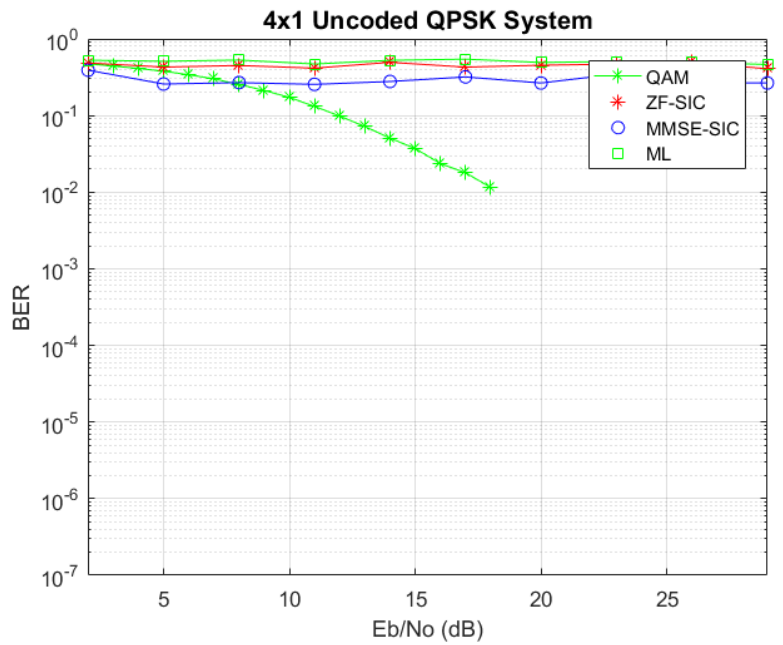

Fig 6-SNR Vs. BER plot for $(4 \times 1)$ MISO system

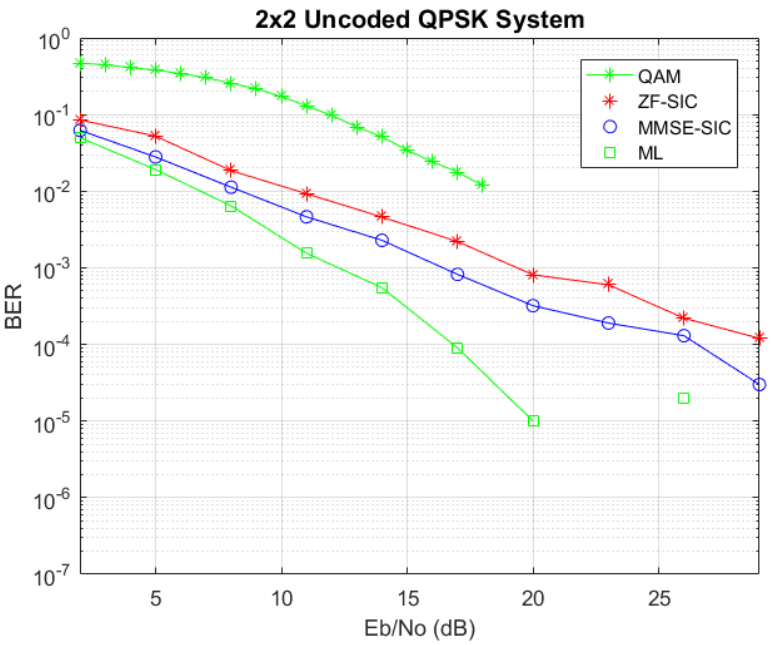

Fig 7-SNR Vs. BER plot for $(2 \times 2)$ MIMO system 


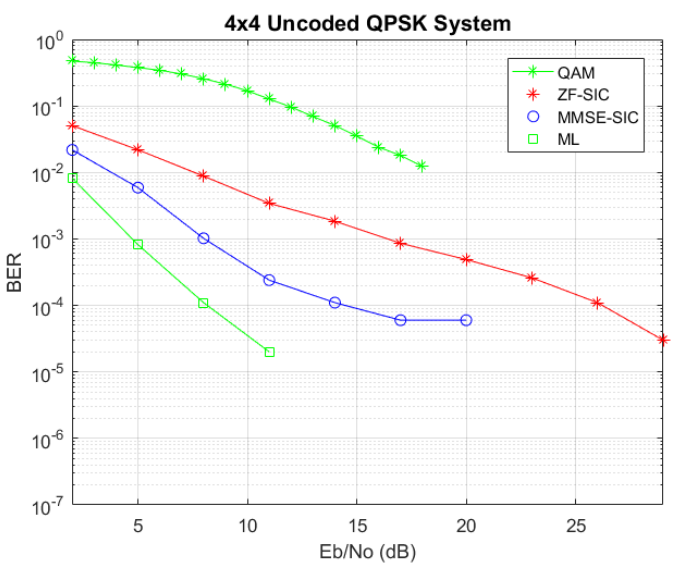

Fig 8-SNR Vs. BER plot for $(4 \times 4)$ MIMO system

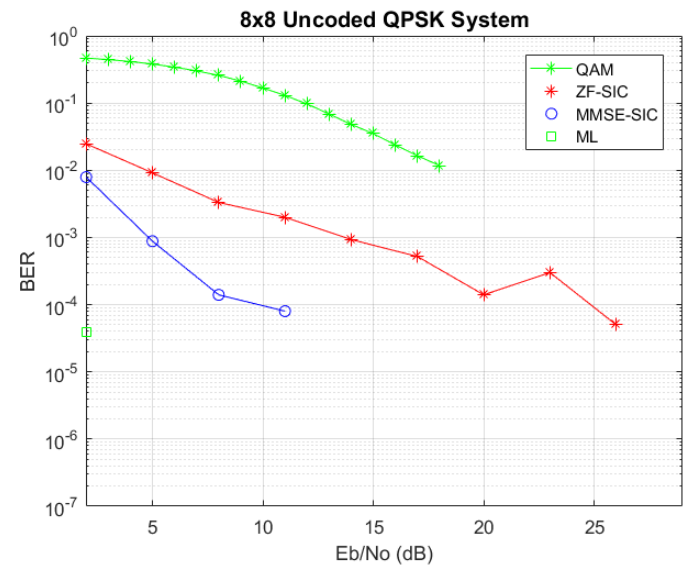

Fig 9-SNR Vs. BER plot for $(8 \times 8)$ MIMO system

\section{CONCLUSIONS AND FUTURE SCOPE}

In this paper, we have discussed the V-BLAST algorithm in details for MIMO-OFDM systems. MIMO system gives better SNR, increased capacity, more data rates and high spectral efficiency. The graphs obtained from simulations show the SNR performances for QAM, ZF/VBLAST, and MMSE/VBLSDT ML/VBLAST detection systems. Except for QAM, all detection methods show better performance for MIMO systems. Out of MMSE, ZF and ML systems the ML/VBLAST has at least 100 times better SNR. Also, we can see that from $(2 \times 2),(4 \times 4)$ and $(8 \times 8)$ MIMO , $(8 \times 8)$ MIMO shows better performance in terms of SNR value and capacity of handling simultaneous channels. Thus as the number of antennas at transmitter and receiver end SNR performance increases with increased capacity. From simulation results, we can conclude that as the number of antennas increased at the transmitter as well as at receiver end, SNR increases rapidly for MIMO systems.

In this paper, performance of SNR is observed by varying number of antennas at Tx and Rx side for AWGN channel. In future we can vary length of bits, change modulation order or use different modulation technique and observe the behavior of SNR.

\section{REFERENCES}

[1] Abdessalem Trimeche, Nesrine Boukid, Anis Sakly, Abdellatif Mtibaa, "Performance Analysis of $\mathrm{ZF}$ and MMSE EqualizerSfor MIMO Systems" IEEE, Design \& Technology of Integrated Systems in Nanoscale Era, 2012.
[2] Bhasker Gupta and Davinder S.Saini," BER Performance Improvement in MIMO Systems Using Various Equalization Techniques" IEEE , Parallel Distributed and Grid Computing (PDGC), 2nd IEEE International Conference 2012.

[3] Amit Grover,Neeti Grover, Keshav Kumar," A Comparative Analysis in Equalization" IEEE,Emerging Trends in VLSI, Embedded System, Nano Electronics and Telecommunication System (ICEVENT), 2013.

[4] P. W. Wolniansky, G. J. Foschini ; G. D. Golden , R. A Valenzuela. "V-BLAST: An architecture for realizing very high data rates over the rich-scattering wireless channel." Signals, Systems, and Electronics, 1998.

[5] Anubhuti Khare, Manish Saxena, Vijendra Singh Mandloi, "Performance Analysis of V-Blast Based MIMO-OFDM System with Various Detection Techniques." IJEAT, ISSN 2249 - 8958, Volume-1, Issue-2, 2011.

[6] Mincheol Park,Brian D. Woerner, Chair William H. Tranter Jeffrey H. Reed," Performance Evaluation of Multiuser Detectors with V-BLAST to MIMO Channel." 2003Virginia.

[7]Ramjee Prasad,"OFDM for wireless communications Sytem",Aalborg University, 2004.

[8] Arsene Pankeu Yomi and Bruce F. Cockburn, " Enhanced MIMO Detection with Parallel V BLAST." IEEE Communications, Computers and Signal Processing (PacRim), 2011.

[9]Ssanjay d deshmukh, Rishi bagadia, Prachi kapoor, Dharmesh gohil," Ofdm-Mimo And V-Blast Algorithm-Key To High Speed Wireless Communication." IJECET volume 4, issue 2, pp. 426-440, 2013

[10] N. Boubaker, K. B. Letaief and R. D. Murch," A Low Complexity Multi-carrier BLAST Architecture for Realizing High DataRates Over Dispersive Fading Channels." IEEE Vehicular Technology Conference, 2001

[11] Kumar, Kuldeep, and Manwinder Singh. "A comparison of different detection algorithms in a MIMO system." International Journal of Advanced Engineering Sciences and Technologies 7.2,vol 7, 301-304,2011.

[12] Shreedhar A Joshi1, Dr. Rukmini T S and Dr. Mahesh H M," Analysis of V-BLAST Techniques for MIMO Wireless Channels with different modulation techniques usingLineaand Non Linear Detection" IJCSI , Vol. 1, Issue 1, November 2011.

[13] Ezio Biglieri, Robert Calderbank,Anthony Constantinides , Andrea Goldsmith, Arogyaswami Paulraj, H. Vincent Poor," MIMO Wireless Communication."2007

[14] Nirmalendu Bikas Sinha, R. Bera, M. Mitra," Capacity And V-Blast Techniques For Mimo Wireless Channel.”JATIT Journal of Theoretical and Applied Information Technology,2010.

[15] Dr. Anubhuti Khare, Manish Saxena, Vijendra singh," Performance Analysis of V-Blast Based MIMO-OFDM System with Various Detection Techniques.” IOSR Journal of Engineering,2012.

[16]http://www.radioelectronics.com/info/antennas/mimo/largemassive-mimo-system 